\title{
A Study of Vitamin D Supplementation to Patients with Chronic Diseases Admitted to Ain Shams University Hospital
}

Ahmed Mohamed Bahaa El Din, Raef Malak Botros, Mariam Michel Ayad Grace, Hany Khairy Mansour*

Department of Internal Medicine and Endocrinology, Faculty of Medicine -Ain Shams University

*Corresponding author: Hany Khairy Mansour, Mobile: (+20) 01221167449, Email: hanyemad@ med.asu.edu.eg

\begin{abstract}
Background: Vitamin D deficiency and insufficiency have become a common problem worldwide. Vitamin D has been associated with all causes of mortality in chronic diseases which are significantly associated with a longer hospital stay and poor outcome.

Objectives: We aimed to investigate the role of empirical vitamin D supplementation in hospitalized patients and its relation to the length of stay and outcome of hospitalization.

Study design: Case control study performed on eighty patients admitted to Internal Medicine Department at Ain Shams University Hospital, with acute deterioration of their chronic illness. Two groups of diseases were included, chronic liver diseases (CLD) and congestive heart failure (CHF).

Methods: Twenty patients of each group were given vitamin D 200,000 IU IM within 3 days of admission (Intervention group) and 20 patients of each group (control group) did not receive vitamin D. Patients were sampled for their vitamin D, calcium and phosphorus levels on admission prior to intervention.

Results: CLD and CHF Intervention groups had a non-significant correlation between vitamin D supplementation with length of hospital stay and mortality in comparison with control groups $(\mathrm{P}=1.000)(\mathrm{p}=0.823)$ respectively. On the other hand, we found baseline vitamin D deficiency was an independent predictor of mortality (P value .018).

Conclusions: Vitamin D deficiency is significantly associated with longer hospital stay and poor outcome of hospital admission. Beneficial effect of empirical vitamin D supplementation can't be achieved with single dose vitamin D (200,000 IU) on CHF and CLD hospitalized patients.
\end{abstract}

Keywords: CHF, CLD, Vitamin D supplementation.

\section{INTRODUCTION}

Vitamin D is the sunshine vitamin. During exposure to sunlight 7-dehydrocholesterol in the skin absorbs ultraviolet $\mathrm{B}$ radiation converting it to previtamin D3. Previtamin D3 being thermodynamically unstable isomerizes within a few hours to form vitamin D3 ${ }^{(\mathbf{1})}$. Humans get vitamin D from exposure to sunlight, from their diet, and from dietary supplementation ${ }^{(2)}$. Research carried out during the past two-decades extended the understanding of actions of vitamin $\mathrm{D}$, from regulating calcium and phosphate absorption and bone metabolism to many pleiotropic actions in organs and tissues in the body. Most observational and ecological studies report association of higher serum 25-hydroxyvitamin D $(25(\mathrm{OH}) \mathrm{D})$ concentrations with improved outcomes for several chronic, communicable and noncommunicable diseases $^{(3)}$.

Vitamin D deficiency is a common problem among Egyptian adolescent girls, for whom contributing factors include inadequate sun exposure due to social/cultural factors and insufficient dietary calcium $^{(\mathbf{4})}$. Vitamin D deficiency is rarely considered or treated in critically ill patients, however, recently reported three cases of life-threatening hypocalcemia secondary to vitamin $\mathrm{D}$ deficiency, highlighting potential acute complications. Deficiency of $25(\mathrm{OH}) \mathrm{D}$ prior to hospital admission is a significant predictor of short and long term all cause patient mortality and blood culture positivity in a critically ill patient population $^{(5)}$.

\section{AIM OF STUDY}

To study the effect of vitamin D supplementation on the outcome of hospitalization for patient with CLD patients with CHF, admitted to Ain Shams University Hospitals with acute deterioration of their illness.

\section{PATIENTS AND METHODS}

\section{Patients:}

This case control study included 80 patients with acute deterioration of their illness namely CLD and $\mathrm{CHF}$ and sub-grouped as follows:

- Group 1: 40 patients suffering from chronic liver diseases (most of them were child $\mathrm{C}$ on ChildPugh score).

- Group 1a: intervention group (20 patients of CLD).

- Group1b: control group 20 patients of CLD (control group).

- Group 2: 40 patients suffering from decompensated heart failure (most of patients with ejection fraction $<40 \%$ diagnosed by echocardiogram).

- Group 2a: intervention group 20 patients of CHD.

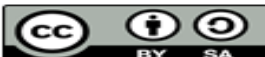

Received:21/7/2020

This article is an open access article distributed under the terms and conditions of the Creative Commons Attribution (CC BY-SA) license (http://creativecommons.org/licenses/by/4.0/) 
- Group 2b: control group 20 patients of CHD.

The intervention group (20 patients from each main group): admitted from April to June 2020 were assigned to be given vitamin D 200.000 IU IM single dose, within first 3 days of admission irrespective of patient's vitamin $\mathrm{D}$ status.

The control group (20 patients from each main group): admitted from January to March 2020 did not receive Vitamin D regardless of their vitamin D status.

\section{Ethical approval and written informed consent:}

An approval of the study was obtained from Ain Shams University academic and ethical committee. Every patient signed an informed written consent for acceptance of the operation.

\section{Inclusion criteria:}

1. Patients of both sexes.

2. Age 21 years old and above.

3. Admitted with acute deterioration of their chronic illness, 40 patients suffering from chronic liver diseases, and 40 patients with heart failure.

\section{Exclusion Criteria:}

Patients admitted with terminal illness were excluded. Patients admitted to surgery or gynecological departments were excluded.

\section{Methodology:}

Blood samples from all participants (control and intervention groups) were withdrawn upon admission and before the injection of vitamin $\mathrm{D}$ in the intervention group. After measurement of vitamin D level, the patients were classified according to their vitamin D status into severe deficiency (vitamin D level $<10 \mathrm{ng} / \mathrm{ml}$ ), deficient group (vitamin D level from 10 to $20 \mathrm{ng} / \mathrm{ml}$ ), insufficient group (vitamin $\mathrm{D} \geq 20<30$ $\mathrm{ng} / \mathrm{ml}$ ) and sufficient group (vitamin D level $>30$ $\mathrm{ng} / \mathrm{ml})^{(6)}$. The outcomes of hospitalization were subsequently compared between the groups and statistically analyzed to determine the effect of vitamin D status or vitamin D supplementation on outcomes.

\section{Statistical analysis}

Data were analyzed using IBM $\odot$ statistics version 23 (IBM@ Corp., Armonk, NY) and MedCalc (C) version 18.2.1 (MedCalc ( $)$ Software bvba, Ostend, Belgium). Continuous numerical variables were presented as mean and SD and inter-group differences were compared using the unpaired t-test. Categorical variables were presented as number and percentage and differences were compared using $\mathrm{Chi}^{2}$ test. The critical P-value was considered statistically significant at $\mathrm{P}<0.05$ and $\mathrm{P}<0.01$ was considered highly significant.

\section{RESULTS}

After adjustment for the disease status and baseline vitamin D status, there was no statistically significant relation between vitamin D supplementation and mortality. However severe vitamin D deficiency was an independent predictor of mortality as presented in table 1 .

Table (1): Multivariable binary logistic regression analysis for the relation between vitamin D supplementation and mortality as adjusted for the disease status and baseline severe vitamin D deficiency status.

\begin{tabular}{|l|c|c|c|c|c|}
\hline Variable & Coefficient & Std. Error & P-value & Odds ratio & 95\% CI \\
\hline CHF (=1) & 0.727 & 0.923 & 0.431 & 2.068 & 0.339 to 12.623 \\
\hline Vitamin D supplementation (=1) & -0.926 & 0.912 & 0.310 & 0.396 & 0.066 to 2.368 \\
\hline $\begin{array}{l}\text { Baseline severe vitamin D } \\
\text { deficiency (=1) }\end{array}$ & 2.134 & 0.953 & $\mathbf{0 . 0 2 5}$ & 8.450 & 1.305 to 54.705 \\
\hline Constant & -3.383 & 0.970 & 0.001 & & \\
\hline
\end{tabular}

After adjustment for the disease status and baseline vitamin D status, there was no statistically significant relation between vitamin D supplementation and mortality. However base line vitamin D level was an independent predictor of mortality as presented in table 2 .

Table (2): Multivariable binary logistic regression analysis for the relation between vitamin D supplementation and mortality as adjusted for the disease status and baseline vitamin D level

\begin{tabular}{|c|c|c|c|c|c|}
\hline Variable & $\begin{array}{c}\text { Coefficien } \\
t\end{array}$ & $\begin{array}{c}\text { Std. Err } \\
\text { or }\end{array}$ & $\begin{array}{c}\text { P- } \\
\text { value }\end{array}$ & $\begin{array}{c}\text { Odds rati } \\
0\end{array}$ & $95 \% \mathrm{CI}$ \\
\hline $\mathrm{CHF}(=1)$ & 0.485 & 0.955 & $>0.05$ & 1.624 & $\begin{array}{c}0.250 \text { to } \\
10.550\end{array}$ \\
\hline Vitamin D supplementation $(=1)$ & -1.047 & 0.918 & $>0.05$ & 0.351 & $\begin{array}{c}0.058 \text { to } \\
2.122\end{array}$ \\
\hline Baseline vitamin D level (ng/ml) & -0.142 & 0.060 & 0.018 & 0.868 & $\begin{array}{c}0.772 \text { to } \\
0.976\end{array}$ \\
\hline Constant & -0.257 & 1.168 & $>0.05$ & & \\
\hline
\end{tabular}

On comparing vitamin D deficiency between the CLD control and CLD intervention groups there was a highly significant difference as in table 3 . 
Table (3): Comparison of baseline characteristics of patients with CLD in control and intervention groups

\begin{tabular}{|l|c|c|c|}
\hline \multicolumn{1}{|c|}{ Variable } & \multicolumn{2}{|c|}{ CLD (n=40) } & \\
\hline Vitamin D level (ng/ml) & Control group (n=20) & Intervention group (n=20) & P-value \\
\hline Vitamin D status & $28.1 \pm 2.8$ & $19.6 \pm 4.9$ & $>0.05$ \\
\hline Severe deficiency & & & 0.011 \\
\hline Deficiency & $1(5.0 \%)$ & $10(50.0 \%)$ & \\
\hline Insufficiency & $4(20.0 \%)$ & $3(15.0 \%)$ & \\
\hline Sufficiency & $5(25.0 \%)$ & $1(5.0 \%)$ & \\
\hline Vitamin D deficiency & $10(50.0 \%)$ & $6(30.0 \%)$ & 0.011 \\
\hline No vitamin D deficiency & $15(75.0 \%)$ & $7(35.0 \%)$ & \\
\hline Vitamin D deficiency & $5(25.0 \%)$ & $13(65.0 \%)$ & \\
\hline
\end{tabular}

On comparing outcome between the CLD control and CLD intervention groups there was no statistically significant difference as in table 4 .

Table (4): Comparison of outcome in patients with CLD in control and intervention groups

\begin{tabular}{|l|c|c|c|}
\hline & \multicolumn{2}{|c|}{ CLD (n=40) } & $\begin{array}{c}\text { P- } \\
\text { value }\end{array}$ \\
\hline Variable & Control group (n=20) & Intervention group (n=20) & $>0.05$ \\
\hline Outcome & $16(80.0 \%)$ & $16(80.0 \%)$ & \\
\hline Discharged home to ICU then & $3(15.0 \%)$ & $3(15.0 \%)$ & \\
\hline $\begin{array}{l}\text { Transferred } \\
\text { discharged home }\end{array}$ & $1(5.0 \%)$ & $1(5.0 \%)$ & \\
\hline Died & $19(95.0 \%)$ & $19(95.0 \%)$ & $>0.05$ \\
\hline Survival & $1(5.0 \%)$ & $1(5.0 \%)$ & \\
\hline Survivor & & & \\
\hline Non-survivor & & & \\
\hline
\end{tabular}

On comparing vitamin D deficiency between the CHF control and intervention groups there was no difference as presented in table 5 .

Table (5): Comparison of baseline characteristics of patients with $\mathrm{CHF}$ in intervention group

\begin{tabular}{|l|c|c|c|}
\hline & \multicolumn{2}{|c|}{ CHF(n=40) } & \\
\hline \multicolumn{1}{|c|}{ Variable } & Control group (n=20) & Intervention group (n=20) & P-value \\
\hline Vitamin D level (ng/ml) & $22.3 \pm 1.9$ & $16.1 \pm 1.2$ & $>0.05$ \\
\hline Vitamin D status & & & $>0.05$ \\
\hline Severe deficiency & $6(30.0 \%)$ & $8(40.0 \%)$ & \\
\hline Deficiency & $3(15.0 \%)$ & $5(25.0 \%)$ & \\
\hline Insufficiency & $6(30.0 \%)$ & $4(20.0 \%)$ & \\
\hline Sufficiency & $5(25.0 \%)$ & $3(15.0 \%)$ & $>0.05$ \\
\hline Vitamin D deficiency & & & \\
\hline No vitamin D deficiency & $11(55.0 \%)$ & $7(35.0 \%)$ & \\
\hline vitamin D deficiency & $9(45.0 \%)$ & $13(65.0 \%)$ & \\
\hline
\end{tabular}

On comparing outcome between the CHF control and intervention groups there was no difference as presented in table 6. 
Table (6): Comparison of outcome in patients with $\mathrm{CHF}$ and control group

\begin{tabular}{|l|c|c|c|}
\hline \multicolumn{1}{|c|}{ Variable } & \multicolumn{2}{|c|}{ CHF (n=40) } & \\
\hline Outcome & & Intervention group (n=20) & P-value \\
\hline Discharged home & $15(75.0 \%)$ & $15(75.0 \%)$ & $>0.05$ \\
\hline $\begin{array}{l}\text { Transferred to ICU then } \\
\text { discharged home }\end{array}$ & $2(10.0 \%)$ & $3(15.0 \%)$ & \\
\hline Died & $3(15.0 \%)$ & $2(10.0 \%)$ & \\
\hline Survival & & & $>0.05$ \\
\hline Survivor & $17(85.0 \%)$ & $18(90.0 \%)$ & \\
\hline Non-survivor & $3(15.0 \%)$ & $2(10.0 \%)$ & \\
\hline
\end{tabular}

As regards baseline characteristics there was statistically significant difference between patients with CLD or

$\mathrm{CHF}$ in intervention group as regards hypertension as presented in table 7.

Table (7): Comparison of baseline characteristics of patients with CLD or CHF in intervention group

\begin{tabular}{|l|c|c|c|}
\hline & \multicolumn{2}{|c|}{ Intervention $(\mathbf{n = 4 0})$} & \\
\hline Variable & CLD $(\mathbf{n = 2 0})$ & CHF $(\mathbf{n = 2 0})$ & P-value \\
\hline Age (years) & $57.8 \pm 12.1$ & $62.9 \pm 10.6$ & $>0.05$ \\
\hline Sex $(\mathrm{M} / \mathrm{F})$ & $8 / 12$ & $7 / 13$ & $>0.05$ \\
\hline DM & $15(75.0 \%)$ & $14(70.0 \%)$ & $>0.05$ \\
\hline Hypertension & $3(15.0 \%)$ & $16(80.0 \%)$ & $<0.001$ \\
\hline
\end{tabular}

As regards baseline characteristics there was statistically significant difference between patients with CLD or CHF in control group as regards hypertension as presented in table 8.

Table (8): Comparison of baseline characteristics of patients with CLD or CHF in control group

\begin{tabular}{|l|c|c|c|}
\hline & \multicolumn{2}{|c|}{ Control $(\mathbf{n = 4 0})$} & \\
\hline Variable & CLD $(\mathbf{n = 2 0})$ & CHF $(\mathbf{n = 2 0})$ & P-value \\
\hline Age $($ years $)$ & $61.5 \pm 9.8$ & $64.1 \pm 9.7$ & $>0.05$ \\
\hline Sex $(\mathrm{m} / \mathrm{F})$ & $9 / 11$ & $6 / 14$ & $>0.05$ \\
\hline DM & $15(75.0 \%)$ & $13(65.0 \%)$ & $>0.05$ \\
\hline Hypertension & $4(20.0 \%)$ & $16(80.0 \%)$ & $<0.001$ \\
\hline
\end{tabular}

There was no statistically significant difference between patients with CLD or CHF in control group as regards vitamin D level and status as presented in table 9.

Table (9): Comparison of baseline characteristics of patients with CLD or CHF in control group

\begin{tabular}{|l|c|c|c|}
\hline & \multicolumn{2}{|c|}{ Control (n=40) } & \\
\hline Variable & CLD (n=20) & CHF (n=20) & P-value \\
\hline Vitamin D level (ng/ml) & $28.1 \pm 2.8$ & $22.3 \pm 1.9$ & $>0.05$ \\
\hline Vitamin D status & & & $>0.05$ \\
\hline Severe deficiency & $1(5.0 \%)$ & $6(30.0 \%)$ & \\
\hline Deficiency & $4(20.0 \%)$ & $3(15.0 \%)$ & \\
\hline Insufficiency & $5(25.0 \%)$ & $6(30.0 \%)$ & \\
\hline Sufficiency & $10(50.0 \%)$ & $5(25.0 \%)$ & \\
\hline Vitamin D deficiency & & & $>0.05$ \\
\hline No vitamin D deficiency & $15(75.0 \%)$ & $11(55.0 \%)$ & \\
\hline Vitamin D deficiency & $5(25.0 \%)$ & $9(45.0 \%)$ & \\
\hline
\end{tabular}

There was no statistically significant difference between patients with CLD or CHF in control group as regards outcome as presented in table 10 . 
Table (10): Comparison of outcome in patients with CLD or CHF in control group

\begin{tabular}{|l|c|c|c|}
\hline \multicolumn{1}{|c|}{ Variable } & \multicolumn{2}{c|}{ Control group (n=40) } & \\
\hline Outcome & CLD (n=20) & CHF (n=20) & P-value \\
\hline Discharged home & & & $>0.05$ \\
\hline Transferred to ICU then discharged home & $16(80.0 \%)$ & $15(75 \%)$ & \\
\hline Died & $3(15.0 \%)$ & $2(10.0 \%)$ & \\
\hline Survival & $1(5.0 \%)$ & $3(15.0 \%)$ & \\
\hline Survivor & & & $>0.05$ \\
\hline Non-survivor & $19(95.0 \%)$ & $17(85.0 \%)$ & \\
\hline
\end{tabular}

There was no statistically significant difference between patients with CLD or CHF in intervention group as regards vitamin D status and level as presented in table 11.

Table (11): Comparison of baseline characteristics of patients with CLD or CHF in intervention group

\begin{tabular}{|l|c|c|c|}
\hline & \multicolumn{2}{|c|}{ Intervention $(\mathbf{n}=\mathbf{4 0})$} & \\
\hline Variable & CLD $(\mathbf{n}=\mathbf{2 0})$ & $\mathbf{C H F}(\mathbf{n}=\mathbf{2 0})$ & P-value \\
\hline Vitamin D level (ng/ml) & $19.6 \pm 1.9$ & $16.1 \pm 1.2$ & $>0.05$ \\
\hline Vitamin D status & & & $>0.05$ \\
\hline Severe deficiency & $10(50.0 \%)$ & $8(40.0 \%)$ & \\
\hline Deficiency & $3(15.0 \%)$ & $5(25.0 \%)$ & \\
\hline Insufficiency & $1(5.0 \%)$ & $4(20.0 \%)$ & \\
\hline Sufficiency & $6(30.0 \%)$ & $3(15.0 \%)$ & \\
\hline Vitamin D deficiency & & & $>0.05$ \\
\hline No vitamin D deficiency & $7(35.0 \%)$ & $7(35.0 \%)$ & \\
\hline Vitamin D deficiency & $13(65.0 \%)$ & $13(65.0 \%)$ & \\
\hline
\end{tabular}

There was no statistically significant difference between patients with CLD or CHF in intervention group as regards outcome as presented in table 12 .

Table (12): Comparison of outcome in patients with CLD or CHF in intervention group

\begin{tabular}{|l|c|c|c|}
\hline & \multicolumn{2}{|c|}{ Intervention (n=40) } & \\
\hline Variable & CLD (n=20) & CHF (n=20) & P-value \\
\hline Outcome & & & 0.603 \\
\hline Discharged home & $16(80.0 \%)$ & $15(75.0 \%)$ & \\
\hline Transferred to ICU then discharged home & $3(15.0 \%)$ & $3(15.0 \%)$ & \\
\hline Died & $1(5.0 \%)$ & $2(10.0 \%)$ & \\
\hline Survival & & & 1.000 \\
\hline Survivor & $19(95.0 \%)$ & $18(90.0 \%)$ & \\
\hline Non-survivor & $1(5.0 \%)$ & $2(10.0 \%)$ & \\
\hline
\end{tabular}

\section{DISCUSSION}

Hypovitaminosis D has become a pandemic, being observed in all ethnicities and age groups worldwide. Environmental factors, such as increased air pollution and reduced ultraviolet B (UVB) exposure, as well as lifestyle factors, i.e., decreased outdoor activities and/or poor intake of vitamin D-rich food, are likely involved in the etiology of a dramatic reduction of vitamin D circulating levels ${ }^{(7)}$.

Recent epidemiological studies have demonstrated that an insufficiency of vitamin D $(<30$ $\mathrm{ng} / \mathrm{ml}$ ), affects $50 \%$ of the population worldwide, while 1 billion people show vitamin $\mathrm{D}$ deficiency $(<10$ $\mathrm{ng} / \mathrm{ml}$ ), as per the cutoffs established by the Endocrine Society Clinical Practice Guidelines ${ }^{(\mathbf{8})}$.
Vitamin D increases calcium absorption, however, its deficiency was linked to increased chronic disease risk (cardiovascular disease, diabetes, dementia, etc.) and all-cause mortality ${ }^{(\mathbf{9})}$.

Vitamin D has multiple functions and target organs. Vitamin D and nuclear binding vitamin D receptor (VDR) after binding can influence the expression of many genes; VDR is widely expressed in the kidney, immune cells, bone cells and other cells $^{(\mathbf{1 0})}$. Studies have shown that the levels and activities of vitamin D are closely related to occurrence and development of many chronic conditions, such as malignancies, autoimmune diseases, metabolic disorders and infectious diseases $^{(\mathbf{1 1})}$. 
In our case-control study performed to examine the effect vitamin D supplementation on outcomes of some chronic diseases i.e. (chronic liver disease and heart failure) on patients admitted to ASUH.

A previous work at ASUH conducted on 80 patients; patients suffering from chronic liver diseases, cerebrovascular stroke, COPD and heart failure, investigating the prevalence of vitamin D deficiency on these patients and its impact on the length of their hospital stay and mortality. Results revealed that vitamin D level had a highly significant inverse correlation with length of hospital stay $(\mathrm{p}=<0.001)$. In vitamin D deficient and insufficient groups there was a highly inverse correlation with outcome of hospital admission $(\mathrm{p}=<0.001)$ $(p=<0.001)$ respectively. On comparing outcome of hospital admission with vitamin D state in studied group; it was found that non-survived patients had highly significant lower vitamin D level than survived patients in deficient and insufficient groups with $p$ value $<0.001$ and it was concluded that vitamin $D$ supplementation should be considered in hospitalized patients, with exception of hypercalcemic and hyperphosphatemic patients ${ }^{(5)}$.

In our study we found significant statistically significant difference between the studied groups of which the baseline vitamin D level was an independent predictor of mortality from the start, which can be explained by the effect of vitamin D as a contributor risk factor for the chronic disease. These results in agreement with those of ${ }^{(\mathbf{1 2}-14)}$; they reported that low vitamin D levels on hospital admission are independent risk factor for mortality in critically ill patients. Lee et al. ${ }^{(15)}$ also reported that mortality rate predicted by the simplified acute physiologic scores (SAPS score) was close 3 times higher in vitamin D deficient patients compared to those who were sufficient.

The same was achieved by Kaur's study which revealed significant difference between studied groups as regards vitamin $\mathrm{D}$ status with $\mathrm{P}$ value $0.04^{(\mathbf{1 6})}$.

In our study; after adjustment for the disease status and baseline vitamin D status, there was no statistically significant difference between vitamin D supplementation and mortality. However, baseline vitamin D deficiency was an independent predictor of mortality.

On the other hand, there was no significant relation between vitamin $\mathrm{D}$ supplementation and mortality in our study after adjustment for the disease status and baseline vitamin D status. However baseline vitamin D level was an independent predictor of mortality.

A study by Arteh goes in agreement with our study. It included 118 patients (43 Hepatitis $\mathrm{C}$ patients with cirrhosis; 57 Hepatitis C patients without cirrhosis; 18 non hepatitis $\mathrm{C}$ patients with cirrhosis) and found 109/118 (92.4\%) patients had some degree of vitamin D deficiency and concluded that vitamin $\mathrm{D}$ deficiency is universal in patients with chronic liver disease and at least one third suffer from severe deficiency, and measurement of vitamin D levels should be part of care of cirrhotic patients ${ }^{(17)}$.

A meta-analysis of 32 cohort studies reported a nonlinear association between serum $25(\mathrm{OH})$ and the hazard ratio (HR) for all-cause mortality, with an increase in the HR starting in the $25(\mathrm{OH}) \mathrm{D}$ range of $75-100 \mathrm{nmol} / \mathrm{L}$, becoming significant in the range of $50-74 \mathrm{nmol} / \mathrm{L}$, and increasing to a maximum of 1.9 for people with $25(\mathrm{OH}) \mathrm{D}<25 \mathrm{nmol} / \mathrm{L}^{(\mathbf{1 8})}$.

Manson's study included 5106 participants to study the effect of vitamin D supplementation effect to lower risk of the primary end points of cancer and cardiovascular events and found the major cardiovascular event occurred in 805 participants (396 in vitamin D group and 409 in placebo group with hazard ratio $.97 ; 95 \% \mathrm{CI}, .85$ to 1.12 ; P value .69 ) and concluded that vitamin $\mathrm{D}$ supplementation did not result in a lower incidence of invasive cancer or cardiovascular events than placebo, which goes in agreement with our study ${ }^{(19)}$.

Vitamin D deficiency has been associated with cholestatic liver disease such as primary biliary cirrhosis. Some studies have suggested that cirrhosis can predispose to development of osteoporosis because of altered calcium and vitamin D homeostasis. This comes in agreement with Arteh's study which included one hundred and eighteen patients (43 with hepatitis $\mathrm{C}$ cirrhosis, 57 with hepatitis $\mathrm{C}$ but no cirrhosis and non-hepatitis $\mathrm{C}$ related cirrhosis) and found 109 of 118 patients (92.4\%) had some degree of vitamin $\mathrm{D}$ deficiency with $\mathrm{P}$ value $=0.05$ and logistic regression model for independent risk factors for severe vitamin $\mathrm{D}$ deficiency; $\mathrm{P}$ value 0.0001 and CI 95\%, 2.2-42.8 $8^{(\mathbf{1 7})}$.

Mozaffari's study conducted a systematic literature search to evaluate the effects of vitamin D supplementation on the severity of chronic liver disease; of total 196 articles found, only 7 relevant documents with 518 studied patients were included. The results showed that the level of vitamin D were lower in patients with chronic liver disease. The results showed that vitamin D supplementation may be beneficial for management of liver diseases at least in certain groups of patients. They also showed that vitamin $\mathrm{D}$ deficiency is associated with the severity of liver disease and can be considered a $\mathrm{n}$ independent prognostic parameter in liver diseases ${ }^{(\mathbf{2 0})}$.

Kubesch's study which included 338 patients with advanced liver fibrosis and cirrhosis; overall, 72 patients $(21 \%)$ had severe vitamin D deficiency. However, patients receiving vitamin D supplements 
had significantly higher vitamin D levels compared to patients without supplements ( $\mathrm{P}$ value $<0.0001$ ). The study also revealed association of severe vitamin D deficiency with risk of hepatic decompensation during follow up $(\mathrm{P}$ value $=0.012, \mathrm{OR}=3.25,95 \% \mathrm{CI}=1.3$ $8.2)^{(21)}$.

Although, in our study there was no significant difference in outcome of chronic diseases (whether survival or not and whether discharged or transferred to ICU) as regard whether they received vitamin $\mathrm{D}$ intervention or not with $\mathrm{P}$ value.

Vitamin D supplementation may have a protective effect against risk of heart failure as shown among participants of the RECORD trial (OR 0.75; 95\%CI: 0.58 to 0.97 ) the effect, however, was not confirmed in the subsequent metanalysis of 21 studies $^{(22)}$.

However to gain this effect it needed to be used in large doses or for long duration ${ }^{(7)}$. The use of calcitriol in the treatment of cardiovascular disorders is often associated with the appearance of dosedependent adverse effects, such as hypercalcemia, hypercalciuria, and the formation of calcifications in the parenchyma of various organs ${ }^{(23)}$.

Our study revealed no significant difference between patients who received vitamin D supplementation and who did not receive vitamin D supplementation as regards outcome and survival in patients with CLD and in patients with CHF.

This goes in run with Chowdhury's study which provided a new metanalysis of observational and trial data relating vitamin D preparation and risk of all causes of mortality and confirmed that low vitamin $\mathrm{D}$ to be associated with elevated risk of multiple adverse outcomes, but their analysis provided that vitamin D supplementation did not seem to reduce the risk of chronic diseases and mortality with relative risk $1.04,95 \%$ confidence interval 0.97 to $1.11^{(24)}$.

Risk of high vitamin D levels and its side effect on the heart as described in CopD study, which is a large observational study from Denmark, prospectively observed 247,574 patients for 7 years and the results indicated a $U$ shaped association between vitamin D levels and CV mortality: The cutoff of 25OHVitD linked with the lowest CV mortality rate was $70 \mathrm{nmol} / \mathrm{L}(28 \mathrm{ng} / \mathrm{ml})$, while the risk for $\mathrm{CV}$ mortality was higher for individuals with either the lowest or the highest 25OHVitD levels. In light of this, new calcitriol analogs Paricalcitol and maxacalcitol are selective VDR activators (VDRAs), and are able to reduce calcium absorption and potassium elimination, and to activate selective metabolic pathways have been synthesized ${ }^{(25)}$.

Finally we can conclude that vitamin D supplementation may improve outcome of chronic diseases but further studies may be needed to show the effect of high doses or longer duration of vitamin D therapy on chronically ill patients in improving their vitamin $\mathrm{D}$ status prior to deterioration of their illness and prior to hospitalization, as the vitamin D status can reflect the disease outcome and mortality from chronic diseases.

\section{CONCLUSION}

Vitamin D plays an important role in the etiology of many diseases and studies shows association of insufficient vitamin D status with various diseases mortality rate.

Further studies are needed to identify the role of vitamin D supplementation to outpatients with diversity of study populations, baseline vitamin D status, dose, and mode and duration of vitamin D supplementation to be considered and explore its role in the disease course and mortality prior to hospitalization.

\section{RECOMMENDATIONS}

We recommend increase society awareness of the importance of vitamin $\mathrm{D}$ and its effect on chronic disease prognosis regarding the mortality. Vitamin D supplementation should be considered in CLD and CHF outpatients, with exception of hypercalcemic and hyperphosphatemic patients, as baseline vitamin $\mathrm{D}$ status affects the disease course and mortality prior to disease deterioration and hospitalization.

\section{REFERENCES}

1. Wacker M, Holick M (2013): Sunlight and vitamin D. Dermatoendocrinol., 5(1): 51-108.

2. Holick M, Garabedian M (2006): Vitamin D: photobiology, metabolism, mechanism of action, and clinical applications. In: Favus MJ, ed. Primer on the metabolic bone diseases and disorders of mineral metabolism. $6^{\text {th }}$ ed. Washington, DC: American Society for Bone and Mineral Research, Pp. 129-37.

3. The Journal of Steroid Biochemistry and Molecular Biology (2018). Glycated albumin is a more useful glycation index than $\mathrm{HbAlc}$ for reflecting renal tubulopathy in subjects with early diabetic kidney disease. Diabetes \& Metabolism Journal, 42(3): 215-23.

4. Botros R, Inas M, Sabry R et al. (2015): Vitamin D deficiency among healthy Egyptian females. Endocrinología y Nutrición, 62(7):314-21.

5. Botros R, AbdElsalam M, Bahaaeldin A et al. (2018): Vitamin D status in hospitalized chronically ill patients. J Am Coll Nutr., 37(7): 578-582.

6. Holick M, Binkley N, Bischoff-Ferrari H et al. (2011): Evaluation, treatment, and prevention of vitamin D deficiency: an endocrine society clinical practice guideline, J. Clin. Endocrinol. Metab., 96 (7): 19111930.

7. Caccamo D, Ricca S, Currò M et al. (2018): Health risks of hypovitaminosis $\mathrm{D}$ : a review of new molecular insights. International Journal of Molecular Sciences, 19(3):892-6. 
8. Hoseinzadeh E, Taha P, Wei C et al. (2018): The impact of air pollutants, UV exposure and geographic location on vitamin D deficiency. Food Chem. Toxicol., 113: 241-254.

9. Forrest K, Stuhldreher W (2011): Prevalence and correlates of vitamin D deficiency in US adults. Nutrition Research, 31(1):48-54.

10. Wang H, Chen W, Li D et al. (2017): Vitamin D and chronic diseases. Aging and Disease, 8(3):346.

11. Ahmed S, Mann M, Hobbs A et al. (2015): Effect of oral vitamin D analogs on mortality and cardiovascular outcomes among adults with chronic kidney disease: a meta-analysis. Clinical Kidney Journal, 8(1):41-8.

12. Matthews $L$, Ahmed $Y$, Wilson $K$ et al. (2012): Worsening severity of vitamin D deficiency is associated with increased length of stay, surgical intensive care unit cost, and mortality rate in surgical intensive care unit patients. The American Journal of Surgery, 204(1):37-43.

13. Melamed M, Michos E, Post W et al. (2008): 25hydroxyvitamin D levels and the risk of mortality in the general population. Archives of Internal Medicine, 168(15):1629-37.

14. Moraes R, Friedman G, Wawrzeniak I et al. (2015): Vitamin D deficiency is independently associated with mortality among critically ill patients. Clinics, 70(5):32632.

15. Lee P, Eisman J, Center J (2009): Vitamin D deficiency in critically ill patients. N Engl J Med., 360:1912-4.

16. Kaur J, Ferguson S, Freitas E et al. (2019): Association of vitamin D status with chronic disease risk factors and cognitive dysfunction in 50-70 Year Old Adults. Nutrients, 11(1):141-45.

17. Arteh J, Narra S, Nair S (2010): Prevalence of vitamin $\mathrm{D}$ deficiency in chronic liver disease. Dig Dis Sci., 55: 2624-2628.
18. Garland C, Kim J, Mohr S et al. (2014): Meta-analysis of all-cause mortality according to serum 25hydroxyvitamin D. American Journal of Public Health, 104(8):43-50.

19. Manson J, Cook N, Lee I et al. (2019): Vitamin D supplements and prevention of cancer and cardiovascular disease. New England Journal of Medicine, 380(1):3344.

20. Mozaffari H, Abbasi H, Goshayeshi L et al. (2016): Effect of vitamin D supplementation on chronic liver disease: systematic literature review. Reviews in Clinical Medicine, 4(1): 14-19.

21. Kubesch A, Quenstedt L, Saleh M et al. (2018): Vitamin D deficiency is associated with hepatic decompensation and inflammation in patients with liver cirrhosis: A prospective cohort study. PloS One, 13(11):0207162.

22. Ford J, MacLennan G, Avenell A et al. (2014): RECORD Trial Group. Cardiovascular disease and vitamin D supplementation: trial analysis, systematic review, and meta-analysis. The American Journal of Clinical Nutrition, 100(3):746-55.

23. Trehan $\mathrm{N}$, Afonso L, Levine $\mathrm{D}$ et al. (2017): Vitamin D deficiency, supplementation, and cardiovascular health. Critical Pathways in Cardiology, 16(3):109-18.

24. Chowdhury R, Kunutsor S, Vitezova A et al. (2014): Vitamin D and risk of cause specific death: systematic review and meta-analysis of observational cohort and randomised intervention studies. BMJ., 348:1903-7.

25. Takada I, Makishima M (2015): Therapeutic application of vitamin D receptor ligands: an updated patent review. Expert Opinion on Therapeutic Patents, 25(12):1373-83. 